\title{
PSICOLOGÍA Y FISIOLOGÍA EN LA DOCTRINA PAVLOVIANA DE LOS REFLEJOS
}

Una de las direcciones que se han acreditado mayormente en el terreno de la psicología biológica es la que se origina en la doctrina del gran fisiólogo ruso Ivan $\mathbf{P}$. PAVLOV (1869-1934) quien llevó a cabo una gran cantidad de experimentos a lo largo de casi medio siglo de continuo trabajo, tendiendo en parte, con dicha experimentación, a explicar la conducta psicológica según el comportamiento del sistema nervioso superior, o sea el que controla desde los hemisferios cerebrales el mecanismo fisiopsíquico que se relaciona directamente. con los procesos de la conciencia.

Como puede observarse, la base del planteamiento pavloviano concierne a dos direcciones científicas tan importantes como son la fisiología y la psicología; en cuanto a la primera, sabemos que la experimentación de Pavlov tiene como objeto básico explicar el comportamiento del sistema nervioso superior, y en cuanto a la segunda, o sea el aspecto psicológico que involucra, se origina en el estrecho contacto que establecen tales procesos con la actividad consciente, que es el objetivo primordial de la psicología.

La investigación de Pavlov se reviste de numerosos y superiores merecimientos; el principal es precisamente el hecho de ser una investigación científica rigurosa, basada en incontables experiencias que se efectuaron en las condiciones más difíciles que puedan producirse en el laboratorio para estudiar el comportamiento del sistema nervioso superior; su rigor metódico es perfectamente definido, exhibe una superior solvencia científica y de ningún modo es posible poner en tela de juicio la seriedad y acuciosidad de sus procedimientos experimentales.

Sin embargo, creemos no sucede otro tanto con la interpretación que se ha querido conferir a las teorías pavlovianas, obtenidas aparentemente como una conclusión estricta de su sistema experimental, aunque en realidad se presentan a través de ciertas tesis interpretativas que suelen llegar a extremos ilegítimos, no sólo porque rebasan el límite señalado en el campo de la experimentación misma, sino también y principalmente porque los partidarios de Pavlov se han investido de un espíritu sectario que pretende a toda costa hacer triunfar una tesis materialista que no sólo entronizaría a la doctrina de Pavlov como el sistema psicológico por excelencia, sino acabaría destruyendo cualquier otra expresión psicológica que no se base estrictamente en la experimentación externa. 
Como sabemos, esta experimentación culmina en una sistema de procesos físicos desarrollados en el laboratorio, por medio del cual se tiende a descubrir una causa material de índole cuantitativa y mesurable que se canaliza específicamente en la doctrina de los reflejos, tanto los incondicionados como condicionados, tesis a la cual nos referimos de inmediato para exponerla como subyacente en el fondo de los acontecimientos psicológicos.

De manera principal, el embate materialista se dirige en contra de la filosofía y la psicología especulativas, cuyo carácter infracientífico somos también nosotros los primeros en reconocer, si bien explicando históricamente los motivos de su aparición, tal como lo hemos hecho al hablar de la psicología filosófica y genéricamente precientífica. Pero no solamente se dirige esta interpretación en contra de teorías especulativas y abstractas, sino inclusive se lanza contra la psicología introspectiva, y más ampliamente en contra de toda la psicología tradicional que para nosotros representa nada menos que el fundamento de la psicología básica; también se dirige furiosamente contra la psicología analítica, a la cual considera prototipo de anticientificidad, de subjetivismo, idealismo y otros "ismos" que en parejas ocasiones suelen prodigar los fanáticos del materialismo, sin olvidar - claro está - atribuir a toda psicología no materialista la poca deseable categoría de ser "retrógrada" y actuar como un instrumento ideológico empleado por la burguesía para mantener la opresión del proletariado.

No ahondaremos en esta pugna, que debería mejor remitirse a los terrenos de la política, ni trataremos de efectuar por ahora una crítica a quienes, amparándose en la teoría de Pavlov, se lanzan contra todas las formas científicas y constructivas -aunque no necesariamente materialistas- de la psicología. Menos aún pretenderíamos denigrar a ese gran investigador de la fisiología que fue el Académico Pavlov, a quien no puede atribuirse responsabilidad sobre la interpretación más o menos torcida que se confiere a sus preciosas investigaciones, cuyos méritos están a salvo de toda sospecha, si bien reclaman -igual que cualquiera otra doctrina- el juicio limitativo que las circunscriba en su propio ámbito y evite la perniciosa confusión que surge cuando una teoría es extendida más allá del ámbito que legítimamente le corresponde.

Después de efectuar esta aclaración, observemos en qué consiste el aspecto medular de la teoría fisiológica pavloviana y cuál es su repercusión en el dominio de la psicología.

El principio orgánico del microfenómeno que constituye la base de los procesos nerviosos en el organismo, es la mecánica del arco reflejo. Recordando brevemente en qué consiste dicha unidad neurofisiológica, señalaremos que tiene como base la sinapsis, o sea la unión de las neuronas que trasmiten una excitación de carácter sensitivo hacia los centros nerviosos que forman parte de un 
complejo fisiológico, y a su vez trasmiten la excitación recibida por el conducto neurosensitivo al sistema neuromotor, que la traduce en movimientos reflejos del más diverso orden.

Este mecanismo constituye la base de la neurofisiología; sabemos que se caracteriza en su forma reducida o microfenoménica como arco reflejo, y en su forma extensa o macrofenoménica como acto reflejo, pues consiste precisamente en reflejar una excitación recibida en los órganos aferentes y sensitivos hacia el sistema nervioso central, y trasmitirla al sistema eferente y neuromotor, traducida principalmente en movimientos musculares $\mathrm{y}$-según pretende la escuela pavloviana - también en reacciones psicológicas.

Ahora bien, este reflejo fisiológico no tiene un carácter pasivo; no se trata de un reflejo absoluto, a la manera como se refleja un rayo de luz en el espejo, o como se reflejaría una excitación física en una reacción mecánica. La naturaleza que asume el reflejo fisiológico está expresada adecuadamente en el término que suele emplearse para calificarlo; dicho término es el de respuesta. En efecto, se dice que el organismo promueve diferentes respuestas a la excitación orgánica, que a su vez se designa como: estímulo, excitación, acción, etcétera.

Así, pues, el sistema nervioso actúa a la manera de un diálogo entre las acciones estimulantes y las respuestas reflejas o reflejadas; este "diálogo fisiológico" se efectúa por medio de un "lenguaje natural" que ha deseado explicar la físiología, contando en primera fila con los acuciosos experimentos pavlovianos.

La teoría de Pavlov tiene como punto de partida el acto reflejo, observado en un primer plano sobre los reflejos incondicionados, que consisten en las respuestas otorgadas espontáneamente por el organismo ante la presencia de ciertos agentes estimuladores. Decimos espontáneamente para denotar la reacción producida conforme a la naturaleza biológica del individuo, o sea mediante la disposición natural de los elementos que integran el sistema nervioso superior en los animales superiores, incluyendo - claro está- al hombre mismo.

El ejemplo típico aplicado por Pavlov en la experimentación es el acercamiento de comida a un perro hambriento, lo cual provoca en forma inmediata - o sea incondicionada- conforme a la constitución biológica del animal, la secreción de saliva y jugos gástricos, así como diversas reacciones neuromusculares que integran el cuadro fisiológico correspondientes a esta excitación. Es fácil comprobar que la misma respuesta se produce en el hombre en condiciones análogas, cuando se le hace "agua la boca" o le da "vueltas el estómago", pues se trata de reflejos naturales que pertenecen no solamente a la especie humana, sino también al amplio sector de los animales superiores que cuentan con un sistema nervioso muy desarrollado.

El carácter incondicionado que asume dicha respuesta instintiva, corresponde a la naturaleza que presenta el sistema reflejo como base constitutiva del sistema nervioso, tanto en los animales superiores como en el hombre. Por ello se dice que los reflejos incondicionados constituyen una manifestación del instinto, y re- 
cíprocamente, que los instintos pueden reducirse - de acuerdo con la teoría de Pavlov- a un conjunto de reflejos incondicionados.

Así como el caso que acabamos de citar (se aproxima comida a un perro hambriento) correspondiente al mas clásico de los experimentos pavlovianos, se producen un gran número de otros más que exploran el bagaje instintivo de la especie, aplicándose a los mecanismos fisiológicos primarios que determinan las funciones vitales y permiten la subsistencia de la especie, así como la adaptación al medio y el desarrollo de las diversas funciones biológicas.

Los partidarios de este sistema fisiológico prefieren emplèar el concepto de "reflejo incondicionado", por considerarlo más científico que el de "instinto", sosteniendo que este último se ha rodeado de una atmósfera idealista o metafísica de indescifrable significado. Se trataría, en tal aspecto, de una cuestión terminológica. Sin embargo, frente a este problema debemos señalar que el concepto tradicional de instinto se inviste además con otra característica, que es la representación introspeccional con respecto al funcionamiento de la conciencia, tal como se percibe en la experiencia interna. Si nos atenemos a este significado introspeccional y representativo, el cariz personal que asume por su expresión inmediata el vocablo instinto, no puede ser reemplazado por reflejo incondicionado, y por ello tanto la humanidad en general como los psicólogos en particular, prefieren seguir refiriéndose al instinto del amor, del saber, del vivir, etcétera, a que no al reflejo incondicionado de lo mismo.

El caso del perro hambriento resulta perfectamente ilustrativo de la operabilidad que adquieren los procesos fisiológicos a partir de una cierta excitación que encuentra la respuesta incondicionada en términos de instinto; pero esta operabilidad mecánica pierde fuerza cuando se coliga con las reacciones psíquicas que acompañan a la producción de los reflejos incondicionados en el ser humano, mediante una asociación que vincula estrechamente a la actividad fisiológica con la psicológica, sin que a pesar de ello puedan identificarse plenamente, ni tampoco reducirse una a la otra.

Podemos decir que, a pesar de los múltiples intentos que se han efectuado para explicar al psiquismo mediante una fórmula fisiológica, no ha sido posible -hasta donde nuestro conocimiento nos lo indica- resolver las manifestaciones primarias del inconsciente individual, como por ejemplo, los instintos de vida, conservación, destrucción, etcétera, por medio del sistema de reflejos. El mismo Pavlov reconoce que los individuos pertenecientes a especies superiores de la naturaleza, o sean las que cuentan con un sistema nervioso desarrollado, son portadores de una abundante dotación instintiva que se localiza en su conducta primaria, sin que los actos reflejos puedan explicar la conformación de dicha naturaleza, que tratándose del hombre no es sólo fisiológica, sino también y primordialmente psicológica, pues característica de la humanidad es la acción interconsciente que promueve en los diversos aspectos de su existencia. 
A mayor abundamiento, la producción de los reflejos incondicionados se efectúa - - según lo reconoce Pavlov- de acuerdo con una constitución innata de la cual puede aceptarse que es portadora del acervo instintivo de la especie. Así encontramos, en esta doctrina acendradamente materialista, la restauración de cuando menos dos sistemas pertenecientes a la psicología clásica, inclusive algunas de sus versiones más tradicionalistas, como son la psicología asociativa y la innatista, que adquieren singular refrendo empiriológico en la nueva y documentada versión experimental de la fisiología pavloviana. Por todo ello, consideramos en este primer enfoque sobre los reflejos incondicionados, que no representan la base última de la conducta psicológica, sino constituye lo que son, un sistema explicativo del conductismo psíquico, que está condicionado por la natu- raleza biológica de los individuos, que a su vez trasmiten la constitución integrativa de la especie que se ha formado a través de innumerables generaciones.

\section{II}

Veamos ahora la segunda parte de esta representativa doctrina que se refiere a los reflejos condicionados. Aquí es donde hace intervenir Pavlov al proceso relacionado con los fenómenos psicológicos, puesto que el reflejo incondicionado constituye exclusivamente la manifestación de una realidad biológica que se trasmite a través de las generaciones entre los miembros de una misma especie, mientras que el reflejo condicionado constituye un testimonio directo del aprendizaje individual.

Partiendo del mismo ejemplo que citamos para ilustrar el reflejo incondicionado - siguiendo en todo las enseñanzas de Pavlov- agregaremos un nuevo elemento que acompaña a la estimulación del reflejo incondicionado. Al acercarse la bandeja con comida al animal hambriento, puede prenderse una luz o producirse algún sonido, repetido el suficiente número de veces para que el animal establezca la correspondiente asociación mecánica; la experimentación demuestra que el animal llega a asociar - "condicionar", dicen los ortodoxos- la presentación de la comida, que constituye el excitante primario, con la luz o el sonido que representa el agente secundario. Así, podrá encenderse la luz o producirse el sonido $y, \sin$ que se presente la comida al animal, se obtendrá la respuesta en forma de secreciones glandulares y movimientos musculares, como en el reflejo original frente a la bandeja con alimento. Esta asociación es la que se designa como reflejo condicionado.

La interpretación "psicológica" elemental que deriva de dicho experimento, consiste en suponer que en esas condiciones el animal "aprendió" a que la luz o el sonido -excitantes secundarios- representan a la comida, es decir, constituyen el símbolo o la señal de la misma, y por ello, al eliminar el agente primario, los agentes secundarios bastarán para producir la excitación. 
En esta forma queda demostrada, en su manifestación elemental, la capacidad que poseen los animales superiores para producir movimientos y reacciones aprendidas, por medio de reflejos condicionados que tienen como base a los reflejos incondicionados que comúnmente se designan como "instintos". Estos últimos constituyen -como hemos dicho- el patrimonio biológico que se acumula a través de una prolongadísima evolución en los caracteres de la especie y se trasmiten por medio de la herencia, mientras aquéllos representan el patrimonio individual de los actos que el sujeto aprende a realizar en el curso de su vida.

Así tenemos que el sistema desarrollado por Pavlov para plantear el problema de la psicología desde un punto de vista natural, consiste en partir de los instintos o reflejos incondicionados, que se producen de manera natural en los individuos como consecuencia hereditaria de la especie, para obtener una gama cada vez más numerosa y compleja de reflejos condicionados, que configuran el acèrvo individual de reacciones aprendidas por el sujeto y, por consiguiente, integran el mecanismo físiológico que se relaciona directamente con la conciencia. De acuerdo con la tesis pavloviana, la conciencia misma puede ser interpretada a través de su lenguaje específico, en el nutrido conjunto de acontecimientos que forman los reflejos condicionados en la vida orgánica y psicológica que, según la propia doctrina, son actos regidos por el sistema nervioso superior y pueden explicarse en términos de reflejos condicionados.

Ahora bien, si analizamos el sentido dinámico que representan los reflejos condicionados, concluiremos que se trata efectivamente de una asociación directa entre el agente secundario (que sustituye al primario y produce la reacción de este último, sin satisfacer las mismas condiciones de especificidad) y la propia reacción fisiológica, promovida por el condicionamiento o aprendizaje del estímulo secundario. Es obvio que dicha asociación requiere, aun en los animales inferiores, un cierto grado de acción autónoma, ya que el mecanismo asociativo se traduce en la reacción condicionada que se observa por la vía experimental. Desde este punto de vista, nos parece inobjetable que el reflejo condicionado sea efectivamente un síntoma de la actividad no consciente propiamente dicha, sino biológica - paraconsciente; el mérito de la experimentación pavloviana no es poco, desde el momento que contribuyó poderosamente a comprender por la vía experimental la mecánica que rige esta clase de procesos, complementando en el terreno fisiológico a la antigua psicología asociativa, que se limitaba en todo caso a constatar la reacción externa sin profundizar en sus implicaciones neurofisiológicas, que fue precisamente el campo donde experimentó con mayores resultados el conspicuo académico ruso.

Como puede observarse, el problema que reporta la interpretación de Pavlov a los acontecimientos neurofisiológicos, no reviste mayores implicaciones, tratándose de animales en que la actividad fisiológica predomina sobre la rudimentariedad parapsíquica que se observa en sus diversas reacciones. Según veremos más adelante, el punto álgido del sistema pavloviano se encuentra al nivel de la 
conducta humana, para la cual observaremos hasta qué punto puede aplicarse el criterio fisiologista como base para una explicación psicológica. Pero antes de llegar a tan importante cuestión de nuestra disciplina, señalemos algunos aspectos generales de la experimentación llevada a cabo por Ivan P. Pavlov, en los animales superiores.

\section{III}

La mayor parte del tiempo que dedicó Pavlov a su trabajo de investigación sobre los reflejos, fue canalizado al estudio experimental del sistema nervioso superior en los animales, debido a que es posible experimentar en ellos efectuando toda clase de intervenciones quirúrgicas, modificaciones endógenas y exógenas de variada especie, mediante una serie de condiciones reguladas cuyo cambio voluntario se requiere para consolidar y enriquecer el acervo experimental. Esta posibilidad es muy limitada en los seres humanos, por razones de orden ético, sociologico y jurídico; no es posible despedazar impunemente un grupo de seres humanos, como puede hacerse con animales, de donde la mayor parte de la experimentación fisiológica ha tenido lugar en los indefensos seres.

Junto a la apreciable ventaja que reporta la utilización de animales en la experiencia científica, se encuentra también la desventaja que representa — sobre todo para nuestro análisis - el hecho de no poderse operar en la mente humana, y para decirlo específicamente en términos pavlovianos, sobre los órganos que constituyen el sistema nervioso superior, de igual manera a como se efectúa en los animales. A pesar de ello, es posible efectuar algunas conclusiones e inferencias analógicas cuyo mayor resultado estriba, a nuestro juicio, en demostrar por la estricta vía experimental que la conciencia tiene un sustento material en el sistema nervioso superior, y es producto de la dilatadísima evolución de las especies naturales, con las que se emparenta desde el momento que sus reacciones biológicas y psicológicas - principalmente las primeras- se encuentran como un antecedente evolutivo en los animales superiores, tal como lo demostró con abundante acopio de datos el académico Pavlov.

Sin embargo, existe una gran diferencia en señalar el parentesco que se registra por el lado biológico entre los animales superiores y el hombre, y la abismal diferencia que encontramos entre aquéllas y éste, en el aspecto psicológico. En el primero - similitud biológica- las analogías son muy considerables y en algunos aspectos los animales superan inclusive al hombre en determinadas formas de sensibilidad, en ciertas propiedades físicas -fuerza, destreza y agilidad- así como algunos mecanismos biológicos de defensa y adaptación. A cambio de ello, la superioridad del hombre se manifiesta terminante en el terreno psicológico, donde se registra un distingo tan radical que las mayores posibilidades "psicológicas" que se hubieran encontrado en los animales más evolucionados, constituyen 
apenas una caricatura de los recursos que exhibe el hombre más rudimentario de la prehistoria.

Si comparamos la constitución física de antropoides como el chimpancé o el orangután, con la del ser humano, encontraremos por parte de los primeros algunas ventajas de carácter físico, las cuales obedecen precisamente a que, al poseer una rudimentaria inteligencia, deben efectuar sus mecanismos de adaptación y defensa a base de fuerza física y recursos biológicos naturales, mientras el hombre lo hace empleando su inteligencia, que le permite sustituir con ventaja los reducidos recursos físicos por el amplio reperterio instrumental que ha construido en las incontables manifestaciones de su vida práctica y sobre todo intelectual.

Por ello, la experimentación naturalista de carácter neurofisiológico, cual la del conspicuo Ivan P. Pavlov, debe encontrar forzosamente una seria limitación que corresponde - por lo demás- a la limitación inherente a la naturaleza biológica de los animales; toda la libertad existente, para experimentar a placer sobre ellos, no puede mostrar algo más de lo que ellos mismos son biológicamente capaces de llevar a cabo. Por otra parte, es cierto que Pavlov experimentó también con seres humanos, atendiendo principalmente a la determinabilidad fisiológica que hemos dicho; pero la teoría "psicológica" que de alli ha derivado, difícilmente supera al estudio de la fisiología misma, sin que pueda acreditarse como una doctrina psicológica propiamente dicha. Por ello, nosotros consideramos que la fisiología pavloviana de los reflejos posee una escasa repercusión psicológica, como intentaremos demostrar más adelante.

La mayor enseñanza que hemos obtenido de las conclusiones formuladas por Pavlov, sobre todo de sus experimentos en perros y antropoides, radica en que estos animales poseen un elevado coeficiente de "inteligencia animal", y permite concluir asimismo lo que ha sostenido acertadamente la teoría evolucionista, o sea que la naturaleza es un gigantesco escenario material donde se representa la gran obra de la creación en sus numerosos episodios evolutivos.

Claro está que Pavlov recoge un copiosísimo acervo de datos empíricos y en cierto modo viene a comprobar experimentalmente lo que ya se había dicho sobre la asociación de los instintos a los hábitos - reflejos incondicionados y condicionados- pero no puede trascender el señalamiento de las reacciones básicas que se observan normal y anormalmente en los animales.

Por otra parte, la experiencia directa con dichos seres nos hace estimarlos no sólo como un abundante complejo de reflejos condicionados e incondicionados, sino también como individuos que albergan una fuente efectiva, tal como sucede principalmente con el perro, lo cual mueve a interpretar la naturaleza animal como una especie de "antecedente filogenético" de la naturaleza humana, con ciertos rudimentos de las manifestaciones emotivas y concienciales que son propias de la naturaleza humana. Pero no obstante la rudimentariedad protoconsciente de los animales, creemos que inclusive su conducta resulta irreductible en su 
integridad a un cúmulo de movimientos reflejos, y que existe un sensible factor de actividad "espontánea" que no corresponde al atavismo incondicionado ni la acumulación condicionada de las reacciones, aprendidas.

Las mayores conclusiones específicas que obtiene la experimentación pavloviana indican, en términos generales, que la vida de los animales superiores exhibe una apreciable capacidad de aprendizaje que obedece a la constante producción de reflejos condicionados que se asimilan sobre los reflejos incondicionados. Es fácil encontrar. esta aguda capacidad de aprendizaje animal, no sólo en la vida diaria, sino en sitios tales como zoológicos, circos, "escuelas", etcétera, donde se desenvuelve hasta un grado apreciable la sensibilidad animal.

\section{IV}

La experimentación pavloviana llegó inclusive a provocar artificialmente ciertos estados conflictivos en el sistema nervioso de los animales, lo cual motivó determinadas situaciones de angustia que se calificaron como "neurosis animales". Haciendo variar las condiciones de la experimentación, llegan a generarse dichas neurosis mediante la alternancia de diversos estados de confusión y fatiga, por una parte, recuperación y adaptación, por la otra, de acuerdo con una caracterología fisiológica que se efectúa observando las diversas potencias de acción y reacción en los animales sujetos al experimento.

Por ejemplo, si se introduce en el proceso del reflejo condicionado un factor negativo que represente sorpresa, molestia o dolor para el animal - un golpe, una descarga eléctrica, un susto- interfiriendo con ello algún àcto normalmente positivo, como puede ser el reflejo de la alimentación, se observará inevitablemente la creación de un conflicto, derivado por una parte de la necesidad que tiene el animal de alimentarse, y por la otra, de la dificultad surgida con la interferencia.

En tal caso, se producirá una reacción del sistema nervioso que se manifiesta en forma de "conflicto neurótico", y puede tener diversas manifestaciones, de acuerdo con la naturaleza del animal; Pavlov observó que la "conducta neurótica" es de irritación y nerviosismo, si el tipo del animal es fuerte, o por el contrario, de inhibición y fatiga que derivan al sueño, si el animal es débil. A tal respecto, la teoría de Pavlov se funda en un esquema dinámico que consiste en la asimilación derivada directamente de los reflejos condicionados y la inhibición que se. opone a los mismos, principalmente cuando surge un agente negativo en la expresenta un obstáculo en el proceso normal del reflejo condicionado, el animal o bien a las tendencias adquiridas o reflejos condicionados en él. Por ello, si se presenta un obstáculo en el proceso normal del reflejo condicionado, el animal tratará de salvar la acción de interferencia buscando una solución "inteligente", como puede ser - por ejemplo- un nuevo camino para llegar a donde se encuentra la comida, cuando el camino habitual es interferido. 
En estas condiciones, la capacidad de aprendizaje se manifiesta en el vencimiento de los obstáculos que se presentan al animal, aunque dicha capacidad encuentra un límite que también se ha establecido en forma bastante precisa para cada género, variando relativamente la facultad de aprendizaje según los individuos, y desde luego, de una especie a otra. Esta diferenciación se agudiza, como es bien sabido, en las especies superiores; perros, caballos, monos, etcétera.

Son numerosísimos los intentos que ha ensayado la experimentación animal no sólo con objeto de investigación, sino también con la finalidad práctica de aprovechar los recursos que brinda la capacidad de aprendizaje en los animales. Ateniéndonos a la investigación pura, señalemos que existen incontables casos de observación en todo género de seres animales, inclusive los más rudimentarios, como puede ser la unicelular ameoba proteus, en la cual se observa una primitiva capacidad de "aprendizaje" que realiza el mecanismo fundamental señalado por Pavlov, a saber: la reacción automática (que podriamos decir instintiva) frente a ciertos excitantes que vulneran la sensibilidad del organismo; el aprendizaje consiste en formar un "hábito" a base de experiencias condicionadas, teniendo como base la dotación biológica del organismo, y como fuente de excitación, el medio ambiente que lo rodea.

La mayor extensión experimental que puede obtenerse para observar la conducta animal no refuta, sino al contrario, refrenda la tesis pavloviana donde se afirma la existencia de reacciones adquiridas por el individuo, sobre las posibilidades que posee en su dotación específica. La escuela de Pavlov se caracteriza por el número incontable de los experimentos realizados en el campo de la fisiología animal, principalmente en los animales superiores que poseen un sistema nervioso desarrollado, cuya sede somática se adjudica en la propia escuela a la corteza de los hemisferios cerebrales; es obvio que, siguiendo el mismo punto de vista, encontramos que el animal más evolucionado es el hombre, cuya actividad psicológica no sería más que una superior manifestación de la fisiología evolutiva que se encuentra en todo el ámbito de la naturaleza.

Pavlov encuentra asimismo la base para establecer una tipología elemental en los animales con los que experimenta, basándose en los caracteres congénitos; que a su vez representan la dotación instintiva, hereditaria e incondicionada, de cada animal. Así, llega a distinguir - de análoga manera a como sucede en el hombre- constituciones fuertes y débiles, individuos más o menos sensibles, adaptables, recuperables, etcétera, dentro de los limites que permite cada especie. Influyen en dicha tipologia algunas características congénitas -como las que hemos citado- y otras adquiridas, como la experiencia asimilada por medio de las prácticas reiteradas que se transforman en reflejos condicionados.

Entre muchos otros experimentos que llevó a cabo Pavlov figura el que observa el desarrollo de la vida animal, según se desenvuelva en libertad, o por el contrario, permanezca en cautiverio; la confronta de lo que sucede en ambos casos arroja la conclusión de que los animales aprisionados adquieren una 
experiencia mucho menor que los dejados en libertad, teniendo en cuenta que éstos se enfrentan a situaciones más variables y diversificadas que los primeros. Esta conclusión puede obtenerse inclusive partiendo de las observaciones cotidianas, y forma parte del consenso general que reconoce - principalmente en el hombre- que la abundancia y diversificación de las experiencias repercute en una mayor formatividad del carácter. La experimentación pavloviana demostró que la vida animal registra una condicionalidad similar a la que encontramos en el hombre, o sea que la integración del individuo depende fundamentalmente del desarrollo que se otorgue a las potencialidades innatas mediante la confronta con la realidad que constituye la experiencia.

También la experimentación biosomática produjo abundantes frutos en el sistema fisiológico de Pavlov. Ahí están los estudios glandulares, que permiten la variación, reducción y aumento experimental de las secreciones, e inclusive la extirpación y el injerto de las propias glándulas, lo cual arroja inapreciables datos sobre el comportamiento del sistema endócrino y su influjo en la conducta. También las zonas cerebrales y los diversos órganos del sistema nervioso sirvieron como punto de referencia para observar el efecto correspondiente en la conducta y el temperamento de los animales, lo cual, expresado en lenguaje pavloviano, equivale a capacidad de reacción mediante los mecanismos de defensa y adaptación al medio, equilibrio y desequilibrio entre la excitación y la inhibición, sensaciones y respuestas de variado orden, que a su vez constituyen la experiencia básica, tanto en las condiciones innatas como adquiridas, frente a las reacciones incondicionadas y las condicionadas, según el proceso asociativo que hemos descrito siguiendo en todo las enseñanzas de Ivan P. Pavlov.

Asimismo, el empleo de excitantes naturales y artificiales fue un asunto que preocupó muy extensamente al académico ruso y sus discípulos, la variada dosificación en condiciones distintas, el aumento y reducción de clichos excitantes hasta límites drásticos significaron otras de las contribuciones más importantes en la investigación de Pavlov y su escuela.

También contribuyó el maestro soviético a producir algunas localizaciones de tipo neurotópico, tales como la radicación de ciertos centros psicógenos, para lo cual sirvió en parte la orientación de la escuela frenológica, cuyo problema ha sido, por lo demás, una de las inquietudes dominantes de la neurofisiología y la psicología naturalista de la época moderna.

Deben estimarse como muy valiosos sus estudios sobre el sistema nervioso superior y su radicación en la corteza de los hemisferios cercbrales, donde se supone que existe la "zona de localización" que funciona como centro de las operaciones neurobiológicas que determinan el comportamiento básico, aunque su acción está directamente vinculada al desempeño que tienen otras zonas cerebrales, como la subcortical y central de los propios hemisferios, el cerebro y la médula oblonga, sobre las cuales operó Pavlov durante muchos años. 
arrojando muy considerable luz que contribuyó eficazmente a explicar sus funciones neurofisiológicas.

En síntesis, puede afirmarse que, de acuerdo con las conclusiones aportadas al estudio fisiológico animal por Ivan P. Pavlov y su escuela materialista, la vida consciente y paraconsciente llamada "actividad nerviosa superior" tiene efectivamente su asiento en el sistema neurocerebral; esta doctrina puede considerarse como el refrendo experimental del estrecho paralelismo psicofísico que ya advirtió la psicología y aun la filosofía antiguas, por cuya virtud toda reacción psíquica está acompañada por una concomitante reacción fisiológica que se supone causa material de aquélla y debe ser determinada objetivamente por la ciencia.

Una de las conclusiones que esgrimen con mayor satisfacción los partidarios del fisiologismo psicológico materialista, particularmente la escuela soviética pavloviana, es la aparente reducción obtenida en dicha escuela al explicar experimentalmente la vida consciente en términos de reflejos condicionados, y los reflejos en términos de actividad nerviosa superior. En efecto, existen un buen número de prosélitos materialistas que no se satisfacen con mantener el sistema de Pavlov como la doctrina fisiológica que efectivamante es, sino pretenden convertirla en doctrina explicativa del psiquismo, e inclusive quieren desterrar a toda otra psicología como falta de "cientificidad", con lo cual se comete - a nuestro juicio- una desconsiderada exageración y un evidente sofisma que comentaremos a título conclusivo en nuestras reflexiones sobre esta importante doctrina.

Por principio de cuentas, ante la abundante experimentación fisiológica realizada por Pavlov podemos preguntar hasta qué punto permite inferir científicamente la existencia en los animales de una estructura conductiva-refleja que puede ser equiparable a la conciencia. La conclusión a tal respecto es sumamente pobre, no sólo por la imposibilidad que tienen los animales de manifestar atributos similares a los del ser humano, sino también por la invencible dificultad para reducir la conducta humana a una serie de reflejos condicionados.

Debemos señalar, como testimonio de objetividad histórica, que el propio Pavlov no deseó en un principio ir más allá del límite que la experimentación le autorizaba, y pretendía ostentarse únicamente como fisiólogo, aunque más tarde llegó a suponer que la fisiología representa a la verdadera "psicología científica". Posteriormente han sido numerosos los partidarios que afirman un reductivismo fisiológico por el cual se explicaría la conducta a partir de una creciente superposición de reflejos condicionados, que a su vez encontrarían un nuevo reflejo materialista en los procesos del sistema nervioso superior, que son susceptibles 
de medirse y cuantificarse de acuerdo con los cánones de la fisiología experimental.

El argumento en que se amparan los partidarios de la tesis pavloviana $-y$ que a nuestro criterio constituye un gran sofisma- para presentarla en calidad de una psicología, es el mismo que sirve, en términos generales, a todos aquellos que pugnan por emitir una explicación fisiológica de la psicología, reduciendo los procesos anímicos a una serie de fenómenos biológicos que efectivamente acontecen en paralelo a la vida psíquica y constituyen su basamento naturalista. Por ejemplo, está plenamente demostrado que al producirse un hecho psicológico - digamos, un acto de pensamiento-- acontecen en el sistema nervioso una serie de fenómenos que son en mayor o menor grado determinables por medio de la experimentación. Tal es el caso del electroencefalógrafo, que registra la producción de ondas eléctricas originadas en la corteza cerebral y trasmitidas al exterior, donde se captan por medio de electrodos y se amplifican millones de veces para detener un registro gráfico que es la traducción plástica de las vibraciones electrónico-cerebrales. En la actualidad se cuentan aparatos mucho más sensibles de los que se disponían en la época de Pavlov, y con toda seguridad que la teoría neurofisiológica formulada por el eminente académico ruso, puede encontrar hoy día un verdadero cúmulo de datos experimentales y, por consiguiente, argumentos mucho más amplios para su comprobación, desde el momento que tiene a su disposición un acopio de experiencias incomparablemente superiores a las que se tenían hasta hace tres décadas.

Sin embargo, pese al notable progreso que han sufrido los sistemas biológicos experimentales en las últimas décadas, la apreciación del funcionamiento fisiológico en relación al psicológico, no ha variado sensiblemente; es decir, la posibilidad que la conducta psicológica sea explicable en términos de fisiología experimental, sigue siendo sumamente precaria. La antigua tesis del paralelismo psicofísico continúa siendo válida en su postulado general y posee cada vez mayores argumentos para su demostración; pero es necesario notar que dicha tesis no ha trascendido la concomitancia biopsíquica que, en último término, viene a ser una modernización científica y experimental del antiguo paralelismo enunciado en el ámbito de la psicología clásica, doctrina ésta de carácter filosófico $y$, por consiguiente, condenada a muerte sin recurso de apelación por los materialistas, a pesar de lo cual representa el más importante predecesor de la doctrina científica que, por sus propios méritos, erigió la investigación fisiológica pavloviana.

Existen dos aspectos fundamentales de la psicología que son los más fuertemente agredidos por la escuela del materialismo fisiológico; el primero de ellos es el carácter subjetivo de las referencias fenoménicas, en concordancia con la subjetividad de las vivencias, lo cual obedece probablemente a que el carácter mismo de la introversión se contrapone en apariencia con la naturaleza exteriorizante del método experimental: en efecto, el método introspectivo per- 
manece en el ámbito de la subjetividad y no sólo deja de traducirse en reacciones materiales, sino que permanece en último término como una experiencia inefable, ya que nadie puede expresar de manera fidedigna lo que siente y percibe en su interioridad, y si lo expresa quedará la expresión misma como una realidad completamente distinta de la vivencia introspeccional.

Obsérvese cualquier caso de los que se presentan continuamente, para comprobar esta aseveración; el acto simple de contemplar un objeto material que tenemos frente a nosotros, produce una vivencia que se efectúa por medio de una imagen canalizada a través de los sentidos. Esta imagen primordialmente visual, es recibida por el sistema óptico y trasmitida a los centros nerviosos, de acuerdo con uno proceso debidamente estudiado por la fisiología. Según el punto de vista que defienden los cultivadores de esta ciencia, la percepción del objeto se producc en un mecanismo subjetivo que puede estudiarse experimentalmente, aunque la imagen misma no pueda ser externada en forma elocuente para hacer innecesaria la introspección, cual sería el caso en que por medio de algún hipotético sistema pudiera proyectarse la imagen óptica, recibida en el sistema cerebral del sujeto, sobre una pantalla exterior, análogamente a como sucede con el encefalograma. Pero como esta proyección no es posible - cuando menos todavía - resulta de ahí que la imagen en sí permanece subjetiva y pierde sentido para la experimentación, que aprehende, únicamente los sucedáneos materiales de que nos habla la fisiología de la visión. A pesar de todo, creemos que ningún materialista querría negar radicalmente a la vivencia introspectiva, pues sería tanto como negarse a sí mismo, pero en cambio lanzan el anatema contra cualquier posible psicología que pretenda erigirse a partir del método introspectivo.

Sin embargo, dicha psicología no sólo es posible, sino también su consolidación como ciencia, mediante una sencilla convención que estriba en aceptar como objetivos los datos que arroja la vivencia subjetiva, suponiendo que incidan de manera similar en la sensibilidad de los demás sujetos y sean elaborados análogamente por su cerebro; en virtud de esta convención, decimos a una persona que está junto a nosotros, que tal o cual objeto tiene determinada forma, color, tamaño y demás características que ocurren a nosotros por medio de la sensibilidad. Suponemos que si nuestro acompañante disfruta de una apreciatividad normal, no habrá mayor problema para estar de acuerdo en esta clase de apreciaciones y, a partir del propio acuerdo, se establece todo un mundo de vivencias y correlatos en los cuales apoya la psicología introspectiva su carácter objetivo y estructural, como un sistema de apreciaciones que alcanza el rango de cientificidad, desde el momento que sus tesis pueden verificarse en la experiencia, aunque por distintos métodos del que se emplea en la psicología experimental; esta diferencia no debe sorprender a nadie, ya que se trata de un problema distinto $y$, por consiguiente, reclama un método distinto también. 
En virtud de las consideraciones que hemos efectuado, no podemos estar de acuerdo con la interpretación fisiologista de los problemas psicológicos; creemos, eso sí, que el estudio de la fisiología arroja una luz de inestimable valor para la comprensión de los problemas biológicos que acompañan al mecanismo de la conciencia, integrada como se encuentra por un mundo representativo donde el existir individual arroja la problemática prístina de la psicología. El correlato fisiológico desempeña el papel de un acompañante naturalista, sin poder identificarse con el sentido interno, inexcrutablemente subjetivo, del acaecer vivencial.

La cientificidad de la psicología no radica — según explicamos antes- en la posibilidad de medir, por ejemplo, las ondas encefalográficas o cardiográficas, que si bien acompañan en su calidad de pulso electrónico a la actividad consciente, de ningún modo explican el decurso autónomo del funcionamiento psíquico. No se ha llegado todavía al extremo científico en que la lectura de un encefalograma revele el nivel intelectual del sujeto, y menos aún que por medio de la electrografía o procedimientos similares se reconozca el tipo de pensamientos que se estaban produciendo al tomar la lectura, de suerte que un mismo encefalograma puede corresponder a un individuo en diversas actitudes psíquicas, ya sea que esté meditando sobre un problema de matemáticas o bien deleitándose con una obra de arte, lo que para la psicología básica son cuestiones completamente distintas.

En cambio, el encéfalo-cardiograma (proseguimos citándolo como ejemplo) registra perfectamente la variación en las tonalidades básicas del fisiopsiquismo, como pueden ser una emoción desbordada, un intenso esfuerzo intelectual, decaimientos en el nivel biológico y lesiones en los órganos respectivos, pero no directamente en su naturaleza psicológica, sino en la repercusión fisiológica que necesariamente adquieren con variaciones notorias de potencial cuantitativo; de ahí el estrecho paralelismo a que aludimos antes. Para la psicología se trata, sin embargo, de una diferencia radical que no puede ser soslayada en aras de un burdo materialismo que pretendiera sostener, por ejemplo, que el hecho de la encefalografía ha logrado la explicación científica del pensamiento, ni menos pretender con ello su definitiva superación, y aun la destrucción de todas las demás modalidades y sistemas de la psicología.

Cosa análoga puede afirmarse de los descubrimientos efectuados en el seno de las ciencias naturales, que en varios aspectos han incrementado notablemente el patrimonio del conocimiento neurofisiológico que acompaña a la vida psíquica, tales como el estudio del sistema nervioso superior, las localizaciones cerebrales, encefálicas y medulares, las reacciones en los sistemas neuromuscular y neurovegetativo, y, en términos generales, todo el conocimiento experimental que proporcionan actualmente las ciencias médico-fisiológicas, iluminando con su 
prepotente luz el extenso ámbito de la fisiología, que sigue figurando a título de una actividad paralela a la psicología, pero nosotros creemos que de ningún modo como base autónoma y netamente explicativa para esta última.

El planteamiento radical de la psicología sistemática consiste en explicar el funcionamiento psicológico a partir de sus propias categorías, que poseen en último término una significación introspectiva, aunque no por ello dan la espalda al método extrospectivo, ni tampoco abjuran del carácter científico, y aun experimental, que puede asumirse en un momento dado el estudio psicológico.

Empleando una alegoría para ilustrar nuestro concepto, diremos que, si se compara el problema neuropsicológico y el socioeconómico, la teoría propiamente dicha de la economía viene a ser la que explica con sus propias leyes -entre otros problemas - la situación monetaria que guarda un país partiendo de consideraciones funcionales a la realidad socio-económica en cuanto tal.

Explicar funcionalmente un problema, como puede ser por ejemplo, el proceso inflacionario, está íntimamente relacionado con el volumen de moneda circulante en relación con la garantía que posee, ya en términos de divisas como por la vitalidad y capacidad económica del nismo.

Ahora bien, la alegoría en cuestión consiste en que la explicación del fenómeno conjunto de la economía se relaciona con un proceso material como es la cantidad de papel-moneda circulante; el símil de la teoría materialista se encuentra en una explicación de cómo el papel-moneda se imprime en las prensas bancarias. El conocimiento de este proceso es necesario para explicar la producción de moneda, que también se explica indicando la clase de papel empleado, los procedimientos de impresión, las tintas, diseños, planchas, prensas, etcétera. Este conocimiento puede ser perfeccionado hasta un amplio nivel científico, en el cual descansaría la doctrina materialista. Pero así como la explicación de cómo se imprimen los billetes de banco no llega a la esencia misma del proceso económico, así tampoco la doctrina fisiológica, con todo y el abundante perfeccionamiento que ha obtenido en fechas recientes, llega al aspecto medular del problema psicológico.

Si más extendemos la comparación, encontraremos que así como existen falsificaciones en el proceso material que significa la impresión de papel-moneda, y ésta se produce mediante el suficiente control de los factores técnicos y científicos que participan en el proceso de impresión, así también existe la "falsificación psicológica" que estriba en la producción artificial de la neurosis, tanto en los animales como en el hombre, sujetándolos a una serie de variaciones anormales y artificialmente producidas para generar un estado de apariencia psicopática, que es más bien fisiopática, por cuanto presenta caracteres externos análogos a la neurosis funcional de la psicología, sin tener el inconfundible origen que encontramos en ésta.

$\mathrm{Y}$ así como es posible obtener una reproducción perfecta de la moneda pública mediante el control de sus condiciones materiales de producción, pero 
desde el punto de vista jurídico y sconómico esta moneda no es válida, así también las neurosis artificiales, y el sistema fisiopático que deriva de la fisiología experimental, no hacen más que reproducir artificialmente el aspecto interno del psiquismo, o sea la esf́era concomitante de los procesos fisiológicos que acompañan a la vida psíquica, de análoga manera a como la existencia de moneda es un hecho material derivado del proceso netamente económico.

Entre otros de los argumentos que se pueden señalar para establecer una suficiente diferenciación de los padecimientos psicógenos y los fisiógenos -teniendo en cuenta en estos últimos la artificialidad del procedimiento experimental- está el consistente en que la neurosis psicógena obedece a una serie de conflictos morales, intelectuales, sociales, culturales y, en suma psicológicos, que todos los conocimientos fisiológicos están imposibilitados para explicar, porque hasta ahora el concepto del psiquismo permanece ajeno a la esencialidad radical de la fisiología, y en términos generales, de la doctrina materialista.

Miguel Bueno 